A. Boccuto, Dipartimento di Matematica e Informatica, via Vanvitelli 1, I-06123 Perugia, Italy. email: boccuto@yahoo.it

V. A. Skvortsov, Department of Mathematics, Moscow State University, Moscow 119992, Russia and Institute of Mathematics, University of Bydgoszcz, 85-065 Bydgoszcz, Poland. email: vaskvor2000@yahoo.com

\title{
THE WARD, PERRON AND HENSTOCK-KURZWEIL INTEGRALS WITH RESPECT TO ABSTRACT DERIVATION BASES IN RIESZ SPACES
}

\begin{abstract}
The Ward and Perron integrals, with respect to abstract derivation bases, for Riesz-space-valued functions are introduced. It is shown that they are equivalent to the respectively defined Henstock-Kurzweil integral.
\end{abstract}

\section{Introduction.}

The Henstock-Kurzweil integral for Riesz-space-valued functions was investigated in numerous papers (see, for example, $[10,14,16,17,1,15]$ ). In [2] a version of this integral with respect to a wide class of derivation bases was considered. The Henstock-Kurzweil approach to integration is closely related to the one of Ward (see [21]). In fact the Henstock theory of integration arose from investigation of the Ward integral (see $[6,7,8]$ ). In the real-valued case, the Ward integral is equivalent to the Henstock-Kurzweil integral and both are known to be equivalent to the Perron integral (see $[5,12])$. In this paper we consider suitable extensions to the Riesz-space-valued case of the Ward and Perron integral with respect to a derivation basis. A definition of the Wardtype integral is no problem and the definition we are introducing in Section

Key Words: Henstock-Kurzweil Integral, Perron integral, Ward integral

Mathematical Reviews subject classification: 28A15, 28B05, 28A39, 42C10, 42C05, 46G10

Received by the editors December 21, 2005

Communicated by: Stefan Schwabik

*This work is supported by RFFI 05-01-00206 and NSh 1657.2003.1 
3 is a direct generalization of the corresponding definition for real functions, giving an integral equivalent to the Henstock-Kurzweil integral (with respect to the same basis). The situation is more delicate with the definition of the Perron-type integral. The problem is that the most natural extension of the definition based on the notion of the pointwise upper and lower derivatives does not suit the purpose. So we must use derivatives of another type, so called $(g)$-derivatives, which are defined by a kind of "global" differentiation procedure. With so-defined upper and lower $(g)$-derivatives, we obtain in Section 4 well defined Perron-type integral and prove that it is equivalent to the Ward integral (with respect to the same derivation basis) and hence to the respective Henstock-Kurzweil integral.

Note that this type of integrals can be applied to the problem of recovering, by generalized Fourier formulae, the Riesz-space-valued coefficients of orthogonal series (see [3]).

\section{Preliminaries.}

A derivation basis (or simply a basis) $\mathcal{B}$ in a measure space $(X, \mathcal{M}, \mu)$ is a filter base on the product space $\mathcal{I} \times X$, where $\mathcal{I}$ is a family of measurable subsets of $X$ having positive measure $\mu$ and called generalized intervals or $\mathcal{B}$-intervals. That is, $\mathcal{B}$ is a nonempty collection of subsets of $\mathcal{I} \times X$ so that each $\beta \in \mathcal{B}$ is a set of pairs $(I, x)$, where $I \in \mathcal{I}, x \in X$, and $\mathcal{B}$ has the filter base property: $\emptyset \notin \mathcal{B}$ and for every $\beta_{1}, \beta_{2} \in \mathcal{B}$ there exists $\beta \in \mathcal{B}$ such that $\beta \subset \beta_{1} \cap \beta_{2}$. So each basis is an ordered directed set and the order is given by the "reversed" inclusion. We shall refer to the elements $\beta$ of $\mathcal{B}$ as basis sets. In this paper we shall always suppose that $\mu(X)<+\infty, \mu(I)>0$ whenever $I \in \mathcal{I}$ and that $(I, x) \in \beta$ implies $x \in I$, although it is not the case in the general theory (see $[11,12,18])$. For a set $L \subset X$ and $\beta \in \mathcal{B}$ we write

$$
\beta(L)=\{(I, x) \in \beta: I \subset L\} \text { and } \beta[L]=\{(I, x) \in \beta: x \in L\} .
$$

We shall assume that for any two basis sets $\beta_{1}, \beta_{2} \in \mathcal{B}$ and for any disjoint sets $L_{1}, L_{2}$ there exists $\beta \in \mathcal{B}$ such that $\beta\left[L_{1} \cup L_{2}\right] \subset \beta_{1}\left[L_{1}\right] \cup \beta_{2}\left[L_{2}\right]$.

We shall suppose in this paper that a basis $\mathcal{B}$ ignores no point; i.e., $\beta[\{x\}] \neq$ $\emptyset$ for any $\beta \in \mathcal{B}$ and any $x \in X$. In the case of a topological space $X$ an example of a basis $\mathcal{B}$ which ignores no point is a Vitali basis, i.e. such a basis that for any $x$, for each neighborhood $U(x)$ of $x$ and for every $\beta \in \mathcal{B}$ the set $\{(I, x) \in \beta, I \subset U(x)\}$ is nonempty. The simplest Vitali derivation basis in $\mathbb{R}^{m}$ is the full interval basis. In this case, $\mathcal{I}$ is the set of all $m$-dimensional intervals in $\mathbb{R}^{m}$ and each basis set is defined by a positive function $\delta$ on $\mathbb{R}^{m}$ 
called gage as

$$
\beta_{\delta}=\{(I, x): I \in \mathcal{I}, x \in I \subset U(x, \delta(x))\},
$$

where $U(x, \delta(x))$ is the ball of center $x$ and radius $\delta(x)$. So the full interval basis is the family $\left(\beta_{\delta}\right)_{\delta}$ where $\delta$ runs over the set of all possible gages.

A finite collection $\pi \subset \beta$ is called a $\beta$-partition if, for any distinct elements $\left(I^{\prime}, x^{\prime}\right)$ and $\left(I^{\prime \prime}, x^{\prime \prime}\right)$ in $\pi$, the $\mathcal{B}$-intervals $I^{\prime}$ and $I^{\prime \prime}$ are non-overlapping (i.e., their intersection is a set of measure $\mu$ zero). If a partition $\pi=\left\{\left(I_{i}, x_{i}\right)\right\} \subset$ $\beta(I)$ for some $I \in \mathcal{I}$ is such that $\cup_{i} I_{i}=I$, then we say that $\pi$ is a $\beta$-partition of $I$. We denote by the symbol $\Pi(\beta ; I)$ the totality of all $\beta$-partitions of a generic $\mathcal{B}$-interval $I$. We say that a basis $\mathcal{B}$ has the partitioning property if the following conditions hold: (i) for each finite collection $I_{0}, I_{1}, \ldots, I_{n}$ of $\mathcal{B}$ intervals with $I_{1}, \ldots, I_{n} \subset I_{0}$ the difference $I_{0} \backslash \cup_{i=1}^{n} I_{i}$ can be expressed as a finite union of pairwise non-overlapping $\mathcal{B}$-intervals; (ii) for each $\mathcal{B}$-interval $I$ and for any $\beta \in \mathcal{B}$ there exists $\pi \in \Pi(\beta ; I)$. In the particular case of the full interval basis on $\mathbb{R}$, this property has long been known as the Cousin lemma. For the full interval basis in $\mathbb{R}^{m}$, the partitioning property can also be established without difficulty. But for some bases this property was proved only recently (see [4]), and there are bases for which it is not valid at all or holds true only in some weaker sense as it is in the case of the symmetric approximate basis (see $[13,20]$ ).

We denote by $R$ a Dedekind complete Riesz space (see [9]). We add to $R$ two extra elements, $+\infty$ and $-\infty$, extending ordering and operations in a natural way (see [2]) and denote $\bar{R}=R \bigcup\{+\infty,-\infty\}$. A nonempty set $T \subset R$ is said to be upper bounded if there exists $s_{1} \in R$ such that $s_{1} \geq t$ for all $t \in T$, lower bounded if there exists $s_{2} \in R$ such that $s_{2} \leq t$ for all $t \in T$, bounded if it is both upper and lower bounded. By convention, we will say that the supremum of any not upper bounded nonempty subset of $R$ is $+\infty$ and the infimum of any not lower bounded nonempty subset of $R$ is $-\infty$. Given a net $\left(r_{\eta}\right)_{\eta \in \Lambda}$ in $\bar{R}$, where $(\Lambda, \geq) \neq \emptyset$ is a directed set, we set

$$
\limsup _{\eta} r_{\eta}=\inf _{\eta}\left[\sup _{\zeta \geq \eta} r_{\zeta}\right] \text { and } \liminf _{\eta} r_{\eta}=\sup _{\eta}\left[\inf _{\zeta \geq \eta} r_{\zeta}\right] \text {. }
$$

We say that $\left(r_{\eta}\right)_{\eta}$ order converges (or simply (o)-converges) to $r \in R$ if $r=$ $\limsup _{\eta} r_{\eta}=\liminf _{\eta} r_{\eta}$, and we write $(o) \lim _{\eta \in \Lambda} r_{\eta}=r$. An $(o)$-net $\left(r_{\eta}\right)_{\eta \in \Lambda}$ is a monotone decreasing net of elements of $\bar{R}$, such that $\inf _{\eta \in \Lambda} r_{\eta}=0$. As a particular case of the above definitions we get the notion of $(o)$-convergence of a sequence in $\bar{R}$ and the notion of $(o)$-sequence. Let $\tau: \mathcal{I} \rightarrow R$ be a $\mathcal{B}$-interval function with $R$ being a Dedekind complete Riesz space. 
We say that $\tau$ is subadditive [superadditive or additive] if $\tau\left(I^{\prime} \cup I^{\prime \prime}\right) \leq$ [respectively, $\geq$ or $=] \tau\left(I^{\prime}\right)+\tau\left(I^{\prime \prime}\right)$ whenever $I^{\prime}$ and $I^{\prime \prime}$ are any two nonoverlapping $\mathcal{B}$-intervals.

\section{The Ward Integral.}

In this section we introduce the Ward integral with respect to a basis $\mathcal{B}$ for Riesz-space-valued functions, and we prove that it is equivalent to the Henstock-Kurzweil integral. From now on, we fix a measure space $(X, \mathcal{M}, \mu)$ and a basis $\mathcal{B}$ in it having the partitioning property. We shall always suppose that all the suprema and the sums "along the empty set" are equal to zero.

We recall the definition of the Henstock-Kurzweil integral with respect to a basis introduced in [2, Definition 3.2], (for the real case, see [19, 12]). If $I$ is a fixed $\mathcal{B}$-interval, $f: I \rightarrow R$ and $\pi \equiv\left\{\left(J_{i}, \xi_{i}\right): i=1, \ldots, n\right\}$ is a partition of

$I$, we will call the quantity $\sum_{i=1}^{n} \mu\left(J_{i}\right) f\left(\xi_{i}\right)$ the Riemann sum associated with $\pi$ and will denote it by the symbol $S(f, \pi)$.

Definition 3.1. Let $R$ be a Dedekind complete Riesz space and $L \subset X$ be a $\mathcal{B}$-interval. We say that $f: L \rightarrow R$ is Henstock-Kurzweil integrable with respect to $\mathcal{B}$ ( simply, $H_{\mathcal{B}}$-integrable) on $L$ if there exists an element $Y \in R$ such that

$$
\inf _{\beta \in \mathcal{B}}(\sup \{|S(f, \pi)-Y|: \pi \in \Pi(\beta ; L)\})=0 .
$$

In this case we write $\left(H_{\mathcal{B}}\right) \int_{L} f=Y$.

It is easy to see that the element $Y$ in (1) is uniquely determined. The following properties of the integral can be easily established (see [2, Propositions 3.5 and 3.6$]$ ).

Proposition 3.2. If $L=I \cup J$ where $L, I, J$ are $\mathcal{B}$-intervals, $I$ and $J$ are non-overlapping and $f$ is $H_{\mathcal{B}}$-integrable on $I$ and on $J$, then $f$ is $H_{\mathcal{B}}$-integrable on $L$, too, and

$$
\left(H_{\mathcal{B}}\right) \int_{L} f=\left(H_{\mathcal{B}}\right) \int_{I} f+\left(H_{\mathcal{B}}\right) \int_{J} f .
$$

Proposition 3.3. If $f$ is $H_{\mathcal{B}}$-integrable on a $\mathcal{B}$-interval $L$ and $J \subset L$ is a $\mathcal{B}$-interval, then $f$ is $H_{\mathcal{B}}$-integrable on $J$, too. 
It follows from Propositions 3.2 and 3.3 that for any $H_{\mathcal{B}}$-integrable function $f: L \rightarrow R$, defined in a $\mathcal{B}$-interval $L$, the indefinite $H_{\mathcal{B}}$-integral is defined as an additive $R$-valued $\mathcal{B}$-interval function on the family of all $\mathcal{B}$-intervals $I$ in $L$. We shall denote it by

$$
F(I)=\left(H_{\mathcal{B}}\right) \int_{I} f
$$

We now introduce the Ward integral with respect to the basis $\mathcal{B}$ for functions with values in a Dedekind complete Riesz space.

Definition 3.4. Let $L \subset X$ be a $\mathcal{B}$-interval and let $f: L \rightarrow R$. A superadditive function $H: \mathcal{I} \rightarrow R$ is called a major function of $f$ if

3.4.1) there exists $\beta \in \mathcal{B}$ such that $\mu(I) f(x) \leq H(I)$ whenever $(I, x) \in \beta$.

A subadditive function $K: \mathcal{I} \rightarrow R$ is said to be a minor function of $f$ if

3.4.2) there exists $\beta \in \mathcal{B}$ such that $\mu(I) f(x) \geq K(I)$ whenever $(I, x) \in \beta$.

The following property is essential for the definition of the integral.

Lemma 3.5. If $H$ is a major function of $f$ and $K$ is a minor function of $f$, then $H(I) \geq K(I)$ for each $I \in \mathcal{I}, I \subset L$.

Proof. If $H$ and $K$ are a major and a minor function of $f$ and $\beta_{1}, \beta_{2}$ satisfy 3.4.1), 3.4.2) respectively, then $\beta \subset \beta_{1} \cap \beta_{2}$ satisfies both 3.4.1) and 3.4.2). This implies

$$
H(J) \geq K(J)
$$

whenever $J \in \mathcal{I}$ and $x \in L$ are such that $(J, x) \in \beta$.

Fix arbitrary $I \in \mathcal{I}, I \subset L$, and let $\pi \equiv\left\{\left(J_{i}, \xi_{i}\right): i=1, \ldots, n\right\} \in \Pi(\beta ; I)$. From (2), superadditivity of $H$ and subadditivity of $K$ we get

$$
H(I) \geq \sum_{i=1}^{n} H\left(J_{i}\right) \geq \sum_{i=1}^{n} K\left(J_{i}\right) \geq K(I) .
$$

The above property of the major and minor functions opens the way to the following definition. 
Definition 3.6. A function $f: L \rightarrow R$ is said to be Ward integrable with respect to $\mathcal{B}$ (or simply $W_{\mathcal{B}}$-integrable) on $L$ if $f$ has both major and minor functions and

$$
\inf \{H(L)\}=\sup \{K(L)\} \in R,
$$

where the involved infima and suprema are taken with respect to all major functions $H$ and all minor functions $K$ of $f$ respectively. The common value $Z$ in (3) we call the $W_{\mathcal{B}}$-integral of $f$, and we write $\left(W_{\mathcal{B}}\right) \int_{L} f=Z$.

We shall prove now that $H_{\mathcal{B}}$-integral and $W_{\mathcal{B}}$-integral are equivalent.

Theorem 3.7. For any function $f: L \rightarrow R$ the following are equivalent:

3.7.1) $f$ is $H_{\mathcal{B}}$-integrable on $L$;

3.7.2) $f$ is $W_{\mathcal{B}}$-integrable on $L$.

Moreover, in this case we have $\left(W_{\mathcal{B}}\right) \int_{L} f=\left(H_{\mathcal{B}}\right) \int_{L} f$.

Proof. 3.7.1) $\Longrightarrow$ 3.7.2) By hypothesis, $f$ is $H_{\mathcal{B}}$-integrable. By virtue of the Saks-Henstock Lemma (see [2, Lemma 3.9]) there exists an $(o)$-net $\left(p_{\beta}\right)_{\beta \in \mathcal{B}}$ such that for each $\beta \in \mathcal{B}$

$$
\sum_{i=1}^{n}\left|\mu\left(J_{i}\right) f\left(\xi_{i}\right)-\left(H_{\mathcal{B}}\right) \int_{J_{i}} f\right| \leq p_{\beta}
$$

for each $\pi=\left\{\left(J_{i}, \xi_{i}\right): i=1, \ldots, n\right\} \in \Pi(\beta ; L)$.

For every $\beta \in \mathcal{B}$, define $\chi_{\beta}: \mathcal{I} \rightarrow R$ by setting

$$
\begin{gathered}
\chi_{\beta}(I) \equiv \sup \left\{\sum_{i=1}^{n}\left|\mu\left(J_{i}\right) f\left(\xi_{i}\right)-\left(H_{\mathcal{B}}\right) \int_{J_{i}} f\right|:\right. \\
\left.\left\{\left(J_{i}, \xi_{i}\right): i=1, \ldots, n\right\} \in \Pi(\beta ; I)\right\}, \quad I \in \mathcal{I} .
\end{gathered}
$$

It is easy to check that $\chi_{\beta}$ is nondecreasing (with respect to inclusion) and $\chi_{\beta}(L) \leq p_{\beta}$.

Let $F$ be the indefinite $H_{\mathcal{B}}$-integral of $f$. It is easy to show that $F+\chi_{\beta}$ is a major function and $F-\chi_{\beta}$ is a minor function of $f$. Thus, by Lemma 3.5, for each $\beta \in \mathcal{B}$ we get

$$
\begin{aligned}
& F(L)-p_{\beta} \leq F(L)-\chi_{\beta}(L) \leq \sup \{K(L): K \text { is a minor function of } f\} \\
\leq & \inf \{H(L): H \text { is a major function of } f\} \leq F(L)+\chi_{\beta}(L) \leq F(L)+p_{\beta} .
\end{aligned}
$$


By arbitrariness of $\beta$ we obtain

$$
\begin{aligned}
& \inf \{H(L): H \text { is a major function of } f\} \\
= & \sup \{K(L): K \text { is a minor function of } f\} \\
= & F(L)=\left(H_{\mathcal{B}}\right) \int_{L} f .
\end{aligned}
$$

This completes the first part of the proof.

$3.7 .2) \Longrightarrow 3.7 .1)$ By $W_{\mathcal{B}}$-integrability of $f$, for every $\beta \in \mathcal{B}$ there exist a major function $H_{\beta}$ and a minor function $K_{\beta}$ such that

$$
0 \leq H_{\beta}(L)-K_{\beta}(L) \leq p_{\beta} .
$$

Pick arbitrarily $\pi \equiv\left\{\left(J_{i}, \xi_{i}\right): i=1, \ldots, n\right\} \in \Pi(\beta ; L)$. We have

$$
K_{\beta}(L) \leq \sum_{i=1}^{n} K_{\beta}\left(J_{i}\right) \leq S(f, E) \leq \sum_{i=1}^{n} H_{\beta}\left(J_{i}\right) \leq H_{\beta}(L),
$$

where $S(f, \pi) \equiv \sum_{i=1}^{n} \mu\left(J_{i}\right) f\left(\xi_{i}\right)$ is the involved Riemann sum. Taking the suprema and the infima, we get

$$
\begin{aligned}
K_{\beta}(L) & \leq \inf \{S(f, \pi): \pi \in \Pi(\beta ; L)\} \\
& \leq \sup \{S(f, \pi): \pi \in \Pi(\beta ; L)\} \leq H_{\beta}(L) .
\end{aligned}
$$

Thus, by (5) and (6), we obtain

$$
\begin{aligned}
0 & \leq \sup \{S(f, \pi): \pi \in \Pi(\beta ; L)\}-\inf \{S(f, \pi): \pi \in \Pi(\beta ; L)\} \\
& \leq H_{\beta}(L)-K_{\beta}(L) \leq p_{\beta} .
\end{aligned}
$$

Moreover, we observe that

$$
\begin{aligned}
& (o) \lim _{\beta \in \mathcal{B}}[\sup \{S(f, \pi): \pi \in \Pi(\beta ; L)\}-\inf \{S(f, \pi): \pi \in \Pi(\beta ; L)\}] \\
= & \inf _{\beta \in \mathcal{B}}[\sup \{S(f, \pi): \pi \in \Pi(\beta ; L)\}]-\sup _{\beta \in \mathcal{B}}[\inf \{S(f, \pi): \pi \in \Pi(\beta ; L)\}]=0,
\end{aligned}
$$

by virtue of (7). Let

$$
\begin{aligned}
Y & \equiv \inf _{\beta \in \mathcal{B}}[\sup \{S(f, \pi): \pi \in \Pi(\beta ; L)\}]=\sup _{\beta \in \mathcal{B}}[\inf \{S(f, \pi): \pi \in \Pi(\beta ; L)\}] \\
& =(o) \lim _{\beta \in \mathcal{B}}[\sup \{S(f, \pi): \pi \in \Pi(\beta ; L)\}]=(o) \lim _{\beta \in \mathcal{B}}[\inf \{S(f, \pi): \pi \in \Pi(\beta ; L)\}] .
\end{aligned}
$$


There exists an $(o)$-net $\left(w_{\beta}\right)_{\beta \in \mathcal{B}}$ such that $\forall \beta \in \mathcal{B}$ we have

$$
|S(f, \pi)-Y| \leq w_{\beta} \text { for all } \pi \in \Pi(\beta ; L),
$$

and hence

$$
\sup \{|S(f, \pi)-Y|: \pi \in \Pi(\beta ; L)\} \leq w_{\beta} .
$$

Thus $f$ is $H_{\mathcal{B}}$-integrable on $L$. Then, as it is already proved in the first part, the integrals coincide.

\section{The Perron Integral.}

The Perron integral for real-valued functions is defined by means of the pointwise upper and lower derivatives. In the case of a Riesz-space-valued $\mathcal{B}$-interval function $\tau$ those derivatives with respect to a basis $\mathcal{B}$ at a fixed point $x$ can be defined respectively as

$$
\begin{aligned}
& \overline{\tau^{\prime}}(x)=\inf _{\beta \in \mathcal{B}}\left[\sup \left\{\frac{\tau(I)}{\mu(I)}:(I, x) \in \beta[\{x\}]\right\}\right] \text { and } \\
& {\underline{\tau^{\prime}}}^{\prime}(x)=\sup _{\beta \in \mathcal{B}}\left[\inf \left\{\frac{\tau(I)}{\mu(I)}:(I, x) \in \beta[\{x\}]\right\}\right] .
\end{aligned}
$$

Since $\beta[\{x\}] \neq \emptyset$ by the assumption imposed on $\mathcal{B}$, we have $\overline{\tau^{\prime}}(x) \geq \underline{\tau^{\prime}}(x)$ and we can define the derivative $\tau^{\prime}(x)$ of the function $\tau$ at $x$ with respect to $\mathcal{B}$ as the common value $\overline{\tau^{\prime}}(x)=\underline{\tau^{\prime}}(x)$ if this equality holds. Unfortunately derivatives defined in this fashion cannot be used in the definition of a Perrontype integral in this case. The reason is that even in the case of the usual full interval basis, there exists a function $\tau$ defined on the family of all intervals of the real line with values in the space $L^{0}([0,1])$ for which the condition $\tau^{\prime}(x)=0$ everywhere on an interval $I=[0,1]$ does not imply that $\tau(I)=0$. This follows from an example given in [1, Remark 3.15].

However a Perron-type integral can be defined for the Riesz-space-valued case if we use another type of derivatives in place of the pointwise ones. Namely, the notions of upper and lower $(g)$-derivatives will suit our purpose. From now on, let $L$ be a fixed $\mathcal{B}$-interval.

Definition 4.1. We say that a $\mathcal{B}$-interval function $\tau: \mathcal{I} \rightarrow R$ is $(g)$-differentiable in $L$ (with respect to the basis $\mathcal{B}$ ) if there exists a function $D \tau: L \rightarrow R$ such that

$$
\inf _{\beta \in \mathcal{B}}\left[\sup \left\{\left|\frac{\tau(I)}{\mu(I)}-D \tau(x)\right|:(I, x) \in \beta[L]\right\}\right]=0
$$


or, equivalently, if there exist a function $D \tau: L \rightarrow R$ and an $(o)$-net $\left(p_{\beta}\right)_{\beta \in \mathcal{B}}$ such that, for all $\beta \in \mathcal{B}$ and for every $(I, x) \in \beta[L]$, we get

$$
|\tau(I)-\mu(I) D \tau(x)| \leq \mu(I) p_{\beta} .
$$

The function $D \tau$ in (8) is called the $(g)$-derivative of $\tau$ in $L$.

Definition 4.2. Let $\tau$ be a $\mathcal{B}$-interval function. The function $\bar{D} \tau: L \rightarrow R$ is said to be upper $(g)$-derivative of $\tau$ in $L$ (with respect to the basis $\mathcal{B}$ ) if there exists an $(o)$-net $\left(p_{\beta}\right)_{\beta \in \mathcal{B}}$ such that for each $\beta \in \mathcal{B}$ and $x \in L$ we have

$$
0 \leq \sup \left\{\frac{\tau(I)}{\mu(I)}:(I, x) \in \beta\right\}-\bar{D} \tau(x) \leq p_{\beta} .
$$

Similarly, the function $\underline{D} \tau: L \rightarrow R$ is said to be lower $(g)$-derivative of $\tau$ in $L$ (with respect to the basis $\mathcal{B}$ ) if there exists an $(o)$-net $\left(q_{\beta}\right)_{\beta \in \mathcal{B}}$ such that for each $\beta \in \mathcal{B}$ and $x \in L$ we have

$$
0 \leq \underline{D} \tau(x)-\inf \left\{\frac{\tau(I)}{\mu(I)}:(I, x) \in \beta\right\} \leq q_{\beta} .
$$

Remark 4.3. Note that for any $\beta \in \mathcal{B}$ and $x \in L$ we get

$$
\begin{aligned}
\underline{D} \tau(x)-q_{\beta} & \leq \inf \left\{\frac{\tau(I)}{\mu(I)}:(I, x) \in \beta\right\} \\
& \leq \sup \left\{\frac{\tau(I)}{\mu(I)}:(I, x) \in \beta\right\} \leq \bar{D} \tau(x)+p_{\beta} .
\end{aligned}
$$

So from arbitrariness of $\beta \in \mathcal{B}$ it follows that $\underline{D} \tau(x) \leq \bar{D} \tau(x)$ for all $x \in L$. Moreover, it is easy to see that the $(g)$-derivative and the upper and lower $(g)$-derivatives are defined uniquely.

Theorem 4.4. A function $\tau: \mathcal{I} \rightarrow R$ is $(g)$-differentiable in $L$ if and only if

$$
\bar{D} \tau(x)=\underline{D} \tau(x) \text { for all } x \in L,
$$

and in this case we get

$$
D \tau(x)=\bar{D} \tau(x)=\underline{D} \tau(x), x \in L .
$$


Proof. If $\tau$ is $(g)$-differentiable in $L$, then there exists an $(o)$-net $\left(w_{\beta}\right)_{\beta \in \mathcal{B}}$ such that, for each $\beta \in \mathcal{B}, x \in L$ and $(I, x) \in \beta$, we have

$$
-w_{\beta} \leq \frac{\tau(I)}{\mu(I)}-D \tau(x) \leq w_{\beta}
$$

and hence, for each $\beta \in \mathcal{B}$ and $x \in L$,

$$
-w_{\beta}+D \tau(x) \leq \sup \left\{\frac{\tau(I)}{\mu(I)}:(I, x) \in \beta\right\}
$$

and

$$
\inf \left\{\frac{\tau(I)}{\mu(I)}:(I, x) \in \beta\right\} \leq w_{\beta}+D \tau(x) .
$$

Taking in (13) the (o)-limit as $\beta$ varies in $\mathcal{B}$, we get

$$
\begin{aligned}
D \tau(x) & \leq(o) \lim _{\beta \in \mathcal{B}}\left[\sup \left\{\frac{\tau(I)}{\mu(I)}:(I, x) \in \beta\right\}\right] \\
& =\inf _{\beta \in \mathcal{B}}\left[\sup \left\{\frac{\tau(I)}{\mu(I)}:(I, x) \in \beta\right\}\right] .
\end{aligned}
$$

Hence, for all $x \in L$, we obtain

$$
D \tau(x) \leq \sup \left\{\frac{\tau(I)}{\mu(I)}:(I, x) \in \beta\right\} .
$$

From (12) we get also

$$
\sup \left\{\frac{\tau(I)}{\mu(I)}:(I, x) \in \beta\right\}-D \tau(x) \leq w_{\beta} \text { for } \operatorname{each} \beta \in \mathcal{B}, \text { for all } x \in L .
$$

From (14), (15) and 4.2 it follows that $D \tau(x)=\bar{D} \tau(x)$ for all $x \in L$. Similarly, it is possible to check that $D \tau(x)=\underline{D} \tau(x)$ for all $x \in L$.

Conversely, suppose that (11) holds, and let $D \tau(x)$ be the common value in (11). Let $\left(p_{\beta}\right)_{\beta \in \mathcal{B}}$ and $\left(q_{\beta}\right)_{\beta \in \mathcal{B}}$ be the nets in the definitions of upper and lower $(g)$-derivatives (see (9) and (10)) respectively, and put $v_{\beta} \equiv p_{\beta}+q_{\beta}$ for every $\beta \in \mathcal{B}$, so that $\left(v_{\beta}\right)_{\beta \in \mathcal{B}}$ is an $(o)$-net. Then, whenever $x \in L$ and $(I, x) \in \beta$, we have

$$
\frac{\tau(I)}{\mu(I)}-D \tau(x) \leq v_{\beta} \text { and } \frac{\tau(I)}{\mu(I)}-D \tau(x) \geq-v_{\beta} .
$$

Hence, $\left|\frac{\tau(I)}{\mu(I)}-D \tau(x)\right| \leq v_{\beta}$, whenever $x \in L,(I, x) \in \beta$. So, $\tau$ is $(g)$ differentiable in $L$, with $D \tau$ being its $(g)$-derivative. 
Theorem 4.5. A function $\tau:[a, b] \rightarrow R$ is $(g)$-differentiable in $L$ if and only if the following Cauchy-type condition holds:

$$
\begin{aligned}
& \text { (o) } \lim _{\beta \in \mathcal{B}}\left[\sup _{x \in L}\left(\sup _{(I, x) \in \beta} \frac{\tau(I)}{\mu(I)}-\inf _{\left(I^{\prime}, x\right) \in \beta} \frac{\tau\left(I^{\prime}\right)}{\mu\left(I^{\prime}\right)}\right)\right] \\
& =\inf _{\beta \in \mathcal{B}}\left[\sup _{x \in L}\left(\sup _{(I, x) \in \beta} \frac{\tau(I)}{\mu(I)}-\inf _{\left(I^{\prime}, x\right) \in \beta} \frac{\tau\left(I^{\prime}\right)}{\mu\left(I^{\prime}\right)}\right)\right]=0 .
\end{aligned}
$$

Proof. Suppose that $\tau$ is $(g)$-differentiable in $L$. Then, by Theorem 4.4, $D \tau$ coincides with $\bar{D} \tau$ and $\underline{D} \tau$. So, by the definitions of upper and lower derivatives, there exist two $(o)$-nets $\left(p_{\beta}\right)_{\beta \in \mathcal{B}},\left(q_{\beta}\right)_{\beta \in \mathcal{B}}$ such that, for every $\beta \in \mathcal{B}$ and $x \in L$, we have

$$
\begin{aligned}
& \sup \left\{\frac{\tau(I)}{\mu(I)}:(I, x) \in \beta\right\}-\inf \left\{\frac{\tau\left(I^{\prime}\right)}{\mu\left(I^{\prime}\right)}:\left(I^{\prime}, x\right) \in \beta\right\} \\
\leq & D \tau(x)+p_{\beta}-D \tau(x)+q_{\beta}=p_{\beta}+q_{\beta} .
\end{aligned}
$$

So the result follows by arbitrariness of $\beta \in \mathcal{B}$ and $x \in L$.

Conversely, let the Cauchy-type condition be satisfied. Put

$$
\begin{aligned}
& \tau^{*}(x)=\sup \left\{\frac{\tau(I)}{\mu(I)}:(I, x) \in \beta\right\}, \\
& \tau_{*}(x)=\inf \left\{\frac{\tau\left(I^{\prime}\right)}{\mu\left(I^{\prime}\right)}:(I, x) \in \beta\right\}, x \in L .
\end{aligned}
$$

So, there exists an $(o)$-net $\left(p_{\beta}\right)_{\beta \in \mathcal{B}}$ such that for each $\beta \in \mathcal{B}$ we get

$$
0 \leq \sup _{x \in L}\left(\tau^{*}(x)-\tau_{*}(x)\right)=\sup _{x \in L}\left(\tau^{*}(x)-\inf \left\{\frac{\tau\left(I^{\prime}\right)}{\mu\left(I^{\prime}\right)}:(I, x) \in \beta\right\}\right) \leq p_{\beta} .
$$

Thus, $\tau^{*}(x)=\underline{D} \tau(x)$ for all $x \in L$. Analogously, we get that $\tau_{*}(x)=\bar{D} \tau(x)$ for all $x \in L$. So, $\bar{D} \tau(x)=\underline{D} \tau(x)$ for all $x \in L$. Hence, by Theorem 4.4, it follows that $\tau$ is $(g)$-differentiable in $L$.

Definition 4.6. Let $f: L \rightarrow R$. A superadditive function $H: \mathcal{I} \rightarrow R$ is called a $(P)$-major function of $f$ if

4.6.1) $\underline{D} H(x) \geq f(x)$ for every $x \in L$.

A subadditive map $K: \mathcal{I} \rightarrow R$ is said to be a $(P)$-minor function of $f$ if 
4.6.2) $\bar{D} K(x) \leq f(x)$ for each $x \in L$.

Definition 4.7. A function $f: L \rightarrow R$ is said to be Perron integrable with respect to $\mathcal{B}$ (or briefly $P_{\mathcal{B}}$-integrable) on $L$ if $f$ has both $(P)$-major and $(P)$ minor functions and

$$
\inf \{H(L)\}=\sup \{K(L)\} \in R,
$$

where the involved infima and suprema are taken with respect to all $(P)$ major functions $H$ and all $(P)$-minor functions $K$ of $f$ respectively. The common value $Y_{p}$ in (16) will be called the $P_{\mathcal{B}}$-integral of $f$, and we write $\left(P_{\mathcal{B}}\right) \int_{L} f=Y_{p}$.

Remark 4.8. We note that, if $H$ is a $(P)$-major function of $f$ and $K$ is a $(P)$-minor function of $f$, then $H(I) \geq K(I)$ for each $I \in \mathcal{I}, I \subset L$. Indeed, let $I \in \mathcal{I}, I \subset L$, and $\pi \equiv\left\{\left(J_{i}, \xi_{i}\right): i=1, \ldots, n\right\} \in \Pi(\beta ; I)$. There exists an (o)-net $\left(p_{\beta}\right)_{\beta \in \mathcal{B}}$ such that, for each $\beta \in \mathcal{B}$ and whenever $x \in L,(I, x) \in \beta$ we have

$$
\begin{aligned}
\frac{K(I)}{\mu(I)}-p_{\beta} & \leq \sup \left\{\frac{K(I)}{\mu(I)}:(I, x) \in \beta\right\}-p_{\beta} \leq f(x) \\
& \leq \inf \left\{\frac{H(I)}{\mu(I)}:(I, x) \in \beta\right\}+p_{\beta} \leq \frac{H(I)}{\mu(I)}+p_{\beta} .
\end{aligned}
$$

Having applied this inequality to each $J_{i}$ we get

$$
\begin{aligned}
& K(I)-\mu(X) p_{\beta} \leq \sum_{i=1}^{n} K\left(J_{i}\right)-\mu(X) p_{\beta} \\
\leq & \sum_{i=1}^{n} H\left(J_{i}\right)+\mu(X) p_{\beta} \leq H(I)+\mu(X) p_{\beta},
\end{aligned}
$$

and so $K(I) \leq H(I)+2 \mu(X) p_{\beta}$ for each $\beta$. Hence $K(I) \leq H(I)$, by arbitrariness of $\beta$.

We now prove the equivalence between the Henstock-Kurzweil, Perron and Ward integrals. In order to do this, it is enough to prove the following.

Theorem 4.9. A function $f: L \rightarrow R$ is $W_{\mathcal{B}}$-integrable if and only if it is $P_{\mathcal{B}}$-integrable, and in this case the two integrals coincide. 
Proof. First of all, we note that any major function $H$ is a $(P)$-major function too, because $f(x) \leq \frac{H(I)}{\mu(I)}$ for any $(I, x) \in \beta$ implies

$$
f(x) \leq \inf \left\{\frac{H(I)}{\mu(I)}:(I, x) \in \beta\right\} \leq \underline{D} H(x)(x \in L) .
$$

Similarly, any minor function is a $(P)$-minor function. So, we obtain that Ward integrability implies Perron integrability, with the same integral value. Conversely, if $H$ is a $(P)$-major function with

$$
f(x) \leq \inf \left\{\frac{H(I)}{\mu(I)}:(I, x) \in \beta\right\}+p_{\beta},
$$

then the map $I \mapsto H(I)+p_{\beta} \mu(I)$ is a major function. In the same way, it is possible to check that, if $K$ is a $(P)$-minor function, then $I \mapsto K(I)-p_{\beta} \mu(I)$ is a minor function. It follows from this that Perron integrability implies Ward integrability.

Combining Theorem 4.9 with Theorem 3.7 we finally get the final assertion of the paper.

Theorem 4.10. Henstock-Kurzweil, Ward and Perron integrals, with respect to a basis $\mathcal{B}$, are equivalent on the class of the Riesz-space-valued functions.

\section{References}

[1] A. Boccuto, Differential and Integral Calculus in Riesz Spaces, Tatra Mountains Math. Publ., 14 (1998), 293-323.

[2] A. Boccuto and V. A. Skvortsov, On Kurzweil-Henstock Type Integrals with Respect to Abstract Derivation Bases for Riesz-space-valued Functions, J. Appl. Funct. Anal,to appear.

[3] A. Boccuto and V. A. Skvortsov, Henstock-Kurzweil Type Integration of Riesz-space-valued Functions and Applications to Walsh Series, Real Analysis Exchange, 29(1) (2003-04), 419-438.

[4] H. Feizić, C. Freiling and D. Rinne, Two-dimensional Partitions, Real Analysis Exchange, 19 (1993-94), 540-546.

[5] R. Henstock, The General Theory of Integration, (1991), Clarendon Press, Oxford. 
[6] R. Henstock, On Ward's Perron-Stieltjes Integral, Canadian J. Math., 9 (1956), 96-109.

[7] R. Henstock, A New Descriptive Definition of the Ward Integral, J. London Math. Soc., 35 (1960), 43-48.

[8] R. Henstock, The Equivalence of Generalized Forms of the Ward, Variational, Denjoy-Stieltjes and Perron-Stieltjes Integrals, Proc. London Math. Soc., 10(3) (1960), 281-303.

[9] W. A. J. Luxemburg and A. C. Zaanen, Riesz Spaces, I, (1971), NorthHolland Publishing Co.

[10] P. McGill, Integration in Vector Lattices, J. Lond. Math. Soc., 11 (1975), $347-360$.

[11] E. J. McShane, A Riemann-type Integral that Includes Lebesgue-Stieltjes, Bochner and Stochastic Integrals, Mem. Amer. Math. Soc., 88 (1969), A. M. S. Providence.

[12] K. M. Ostaszewski, Henstock Integration in the Plane, Mem. Amer. Math. Soc., 353 (1986), A. M. S. Providence.

[13] D. Preiss and B. S. Thomson, The Approximate Symmetric Integral, Can. J. Math., 41 (1989), 508-555.

[14] B. Riečan, On the Kurzweil Integral for Functions with Values in Ordered Spaces, I, Acta Math. Univ. Comenian., 56-57 (1989), 411-424.

[15] B. Riečan and T. Neubrunn, Integral, Measure and Ordering, (1997), Kluwer Acad. Publ.

[16] B. Riečan and M. Vrábelová, On the Kurzweil Integral for Functions with Values in Ordered Spaces, II, Math. Slovaca, 43 (1993), 471-475.

[17] B. Riečan and M. Vrábelová, On the Kurzweil Integral for Functions with Values in Ordered Spaces, III, Tatra Mountains Math. Publ., 8 (1996), 93-100.

[18] V. A. Skvortsov, Variations and Variational Measures in Integration Theory and Some Applications, J. Math. Sciences, 91 (1998), 3293-3322.

[19] B. S. Thomson, Derivation Bases on the Real Line, Real Analysis Exchange, 8 (1, 2) (1982-83), 67-207, 278-442. 
[20] B. S. Thomson, Symmetric Properties of the Functions, Monographs and textbooks in Pure and Appl. Math, 183 (1994), Marcel Dekker.

[21] A. J. Ward, The Perron-Stieltjes Integral, Math. Z., 41 (1936), 578-604. 
